\title{
HOPF-FRIEDRICHS BIFURCATION AND THE HUNTING OF A RAILWAY AXLE*
}

BY

R. R. HUILGOL

The Flinders University of South Australia, Bedford Park, S.A. 5042

\begin{abstract}
After deriving the equations of motion which govern the lateral and yaw motions of a railway axle, these are cast in the form of a system of first-order nonlinear differential equations. To this system the Hopf-Friedrichs bifurcation theory is applied to determine when a periodic orbit will bifurcate from the equilibrium position. Sufficient conditions to guarantee the stability of the orbit are investigated.
\end{abstract}

1. Introduction. Common experience shows that trains sway from side to side and yaw about a vertical axis; quite often these motions are rhythmic in character, especially when a train is running on a straight track. In addition to the above two oscillations, a train bogie (truck) can pitch forward as well and, not surprisingly, these periodic motions have been the subject of many investigations, beginning with Carter [1] in 1922. The underlying reason for the research has been that these oscillations may grow without bounds and hence lead to a derailment of the vehicle. That these fears are well founded becomes clear when one notes that out of a recent batch of locomotives built for Amtrak, a few have been derailed at speeds of around $105 \mathrm{Kph}$.

Now the equations of motion which govern the hunting-the expression commonly used to describe the periodic motions-of a locomotive form a system of coupled secondorder nonlinear differential equations. Therefore, these can be cast quite readily into a system of coupled first-order nonlinear differential equations. Once the physical dimensions, the mass, the springs, the dampers, the wheel and rail profiles of a locomotive and the track are specified, the only parameter which can be altered is the forward speed. Hence the equations of motion can be thought of as a real $n$-dimensional $(n \geq 2)$ firstorder system of autonomous differential equations depending on a real parameter $\Gamma$, namely as

$$
\dot{\mathbf{x}}=\hat{\mathrm{F}}(\mathbf{x}, \mathrm{T}) .
$$

Here $\mathbf{x}$ is the vector denoting the rolling, pitching and yaw motions and $\Gamma$ is the measure of the forward speed. Once the equations are cast in the form (1.1), the mathematical problems are:

(i) does the coupled system exhibit a periodic solution which bifurcates from the equilibrium position, which can be interpreted as steady forward motion with no additional oscillations?

* Received October 7, 1977. The author wishes to thank Dr. V. K. Garg of the American Association of Railways Research Center, Chicago, for bringing the general area of locomotive hunting to his attention and for many fruitful discussions. 
(ii) if the periodic motion exists, is it stable or unstable?

These questions are relatively easy to answer because of the pioneering work of $\mathrm{E}$. Hopf [2] in 1942 in which he discussed the bifurcation of periodic solutions of (1.1) under the assumption that $\mathrm{F}$ is analytic in the neighborhood of a critical point $\left(\mathrm{a}^{0}, \mathrm{r}_{0}\right)$. Friedrichs [3] was able to derive an identical existence result for (1.1), when $n=2$, under the assumption that $\mathbf{F}$ is only three times continuously differentiable. Recently, the extension of Friedrichs' proof to the case $n>2$ have been made by Poore [4] and Marsden and McCracken [5]. The approach by Poore [4] is based on the alternative theory developed by Cesari [6] and Hale [7] and needs $\hat{F} \in C^{3}$ in its proof, while that of Marsden and McCracken [5] makes use of the center manifold theorem and demands $\hat{F} \in C^{4}$ in the proof. In [4] and [5], after the existence of a bifurcating periodic solution to (1.1) has been established, the stability of the periodic orbit is also studied and sufficient conditions guaranteeing the stability are also given. The above results are local in character.

The global result, meaning the continuation of a bifurcated periodic orbit, is of physical and mathematical interest as well. A letter from Professor W. F. Langford states that such an investigation was begun by Alexander and Yorke and continued by Ize, Nussbaum, and Chow and Mallet-Paret. The conclusions of the results of Langford, for example, are that the local solutions are part of a continuum of periodic solutions having at least one of the properties:

(i) the amplitude is unbounded;

(ii) the range of the bifurcation parameter is unbounded;

(iii) the period is unbounded;

(iv) a certain inner product takes arbitrarily small values.

With an additional hypothesis, (iv) reduces to the statement that the continuum joins another bifurcation point distinct from the first.

Turning to the locomotive hunting problem, it would be of interest to prove that at least one of the above possibilities (i), (iii) or (iv) occur. For as the speed is increased, these would imply that the locomotive would be derailed or that the oscillations die out or that the oscillations would hunt between two speeds, or a combination of the above three.

In the present investigation, which is the first in a series, a detailed study is made of the hunting of a locomotive axle from the point of view of a local bifurcation of a periodic orbit. Because one is concerned with a single pair of wheels connected by a rigid axle, only lateral and yaw motions are considered and pitching is ignored. The equations of motion governing the lateral and yaw motions used here are those derived by Brann [8] and the reader is referred to that paper for the full details of derivation, though a brief resumé is given here.

2. The governing equations. Let the wheel axle roll at a constant speed $V$ along a straight track. The generalized coordinate $y$ represents the lateral displacement of the center of mass of the system from the center line of the track while the second generalized coordinate $\theta$ measures the yaw motion (see Fig. 1). The equations of motion, for an axle of mass $m$ and moment of intertia $I$, are:

$$
\begin{gathered}
m \ddot{y}+\left\{N_{2}{ }^{\prime}-N_{1}{ }^{\prime}+F_{1}{ }^{\prime}+F_{2}{ }^{\prime}\right\} \cos \theta=0, \\
I \ddot{\theta}+b\left(P_{1}-P_{2}\right)=0 .
\end{gathered}
$$

Here $N_{1}{ }^{\prime}$ and $N_{2}{ }^{\prime}$ are the lateral force components; the vertical reactions are $N_{1}$ and $N_{2}$, 


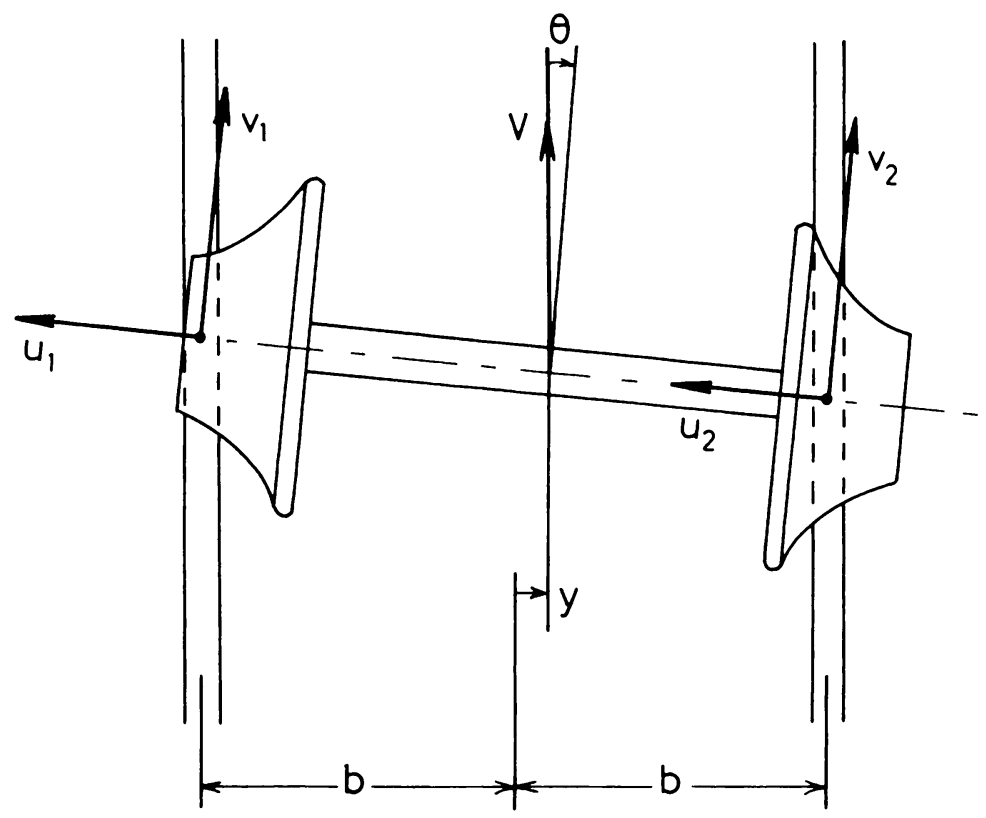

FIG. 1.

each equal to $m g / 2 ; F_{1}{ }^{\prime}$ and $F_{2}{ }^{\prime}$ are the lateral components of the frictional forces, with $P_{1}{ }^{\prime}$ and $P_{2}{ }^{\prime}$ their longitudinal components; $b$ is the semi-gauge of the track. We let

$$
N_{i}{ }^{\prime}=N_{i} \tan \delta_{i}, \quad i=1,2 .
$$

Then

$$
F_{i}{ }^{\prime}=F_{i} \cos \delta_{i}, \quad i=1,2,
$$

as is obvious from Fig. 2. Following Brann [8], we assume that $\left(\tan \delta_{2}-\tan \delta_{1}\right)$ is an odd function of $y$, or

$$
\tan \delta_{2}-\tan \delta_{1}=\sum_{s=0}^{n} e_{s} y^{2 s+1},
$$

where $e_{s}, s=0,1, \ldots, n$, are constants, and that

$$
\cos \delta_{1}+\cos \delta_{2}=k, \quad k<2
$$

where $k$ is a constant very close to 2 .

The equations of motion so far are fairly simple to define and derive. What makes for uncertainty and complexity is the relation of the frictional forces to the weight of the axle and the speed of the train. The formulae relating these quantities were originally introduced by Carter [1,9] and are still used, though a fully nonlinear theory is available and has recently been reviewed by Kalker [10]. In the present paper, a linearized version is employed because we are interested in a local bifurcation; to obtain these linearized formulae relating the frictional forces to the weight and speed, we proceed to define the creep velocities [1] next.

Consider an axle which rolls ahead with a constant speed $V$; as it moves forward, it moves laterally with a speed $\dot{y}$ and yaws with an angular speed $\dot{\theta}$. Focus attention on the 

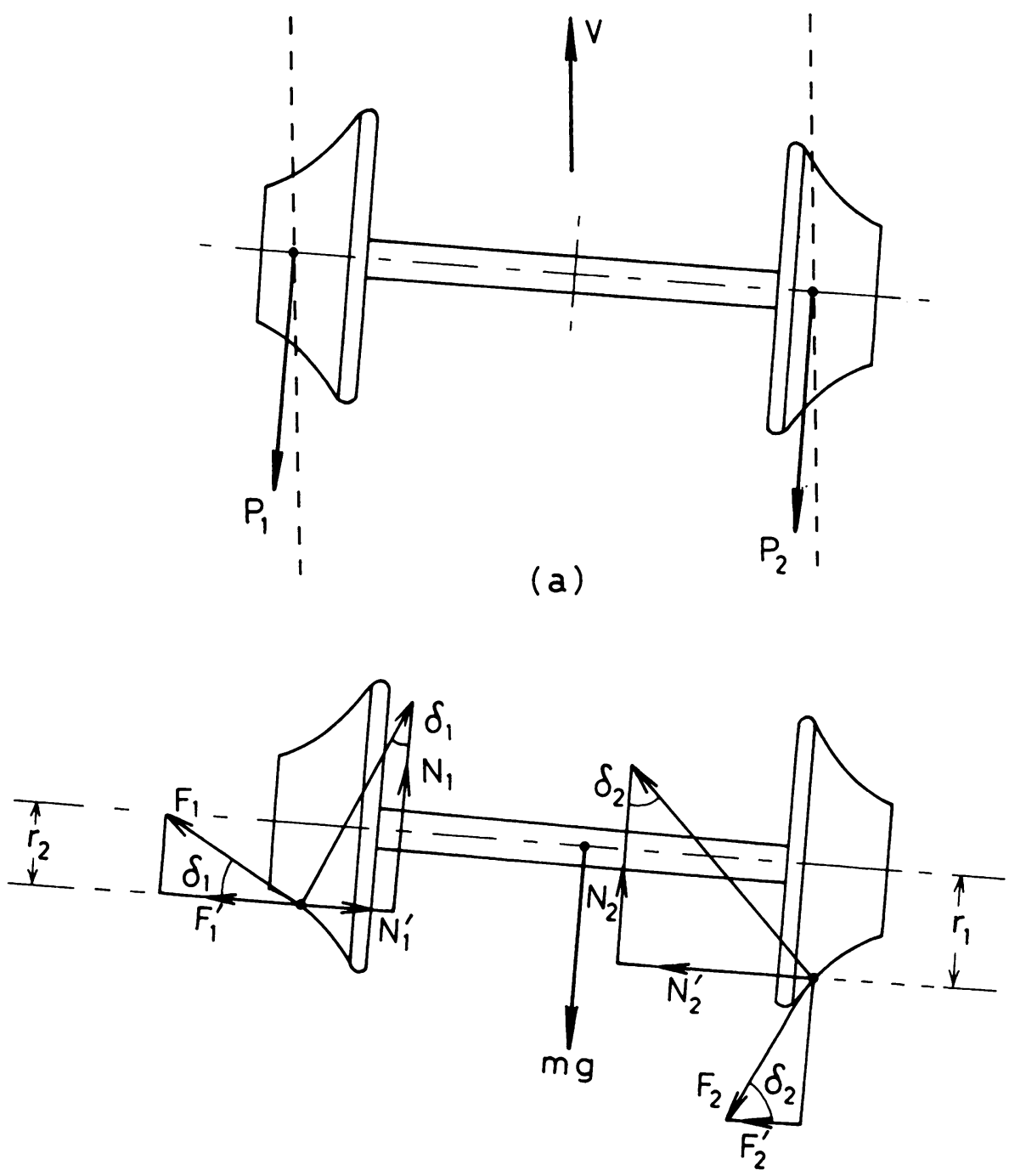

(b)

FIG. 2.

point of contact of each wheel with a rail and observe that if perfect rolling were to occur, the point of contact would be instantaneously at rest. In imperfect rolling, this point would migrate, or creep, over the rail.

To a first approximation, the horizontal creep velocities $v_{1}$ and $v_{2}$ are easy to define. They are given by

$$
\begin{aligned}
& v_{1}=V-\Omega r_{1}+b \dot{\theta}, \\
& v_{2}=V-\Omega r_{2}-b \dot{\theta},
\end{aligned}
$$

where $\Omega$ is the angular velocity of the wheels and $r_{1}$ and $r_{2}$ the respective radii of contact of the wheels with the rails at any instant. 
Similarly, the lateral creep velocities are given, on putting $\sin \theta \doteq \theta$, by

$$
u=u_{1}=u_{2}=\dot{y}-V \theta \text {. }
$$

Following Brann [8], let

$$
\begin{gathered}
F_{1}=F_{2}=\frac{f}{V} u, \\
P_{i}=\frac{f}{V} v_{i}, \quad i=1,2
\end{gathered}
$$

be the frictional forces. Here $f$ is the creep coefficient and is assumed to be a constant. For numerical purposes we shall take $f=m g / 2$, as in [8].

It is clear that, in addition to (2.5), we need an expression for $r_{1}-r_{2}$. Let this be given by:

$$
r_{1}-r_{2}=\sum_{s=0}^{n} q_{s} y^{2 s+1},
$$

where the $q_{s}$ are constants. Note that physical reasoning demands that $r_{1}-r_{2}$ be an odd function of $y$ and this has been used above.

Let a nondimensional variable $z$ be introduced through

$$
z=y / b .
$$

The equations of motion (2.1) and (2.2) now become:

$$
\begin{gathered}
m b \ddot{z}+\frac{f k b}{V} \dot{z}+\frac{m g}{2} \sum_{s=0}^{n} e_{s} z^{2 s+1}=f k \theta, \\
I \ddot{\theta}+\frac{2 f b^{2}}{V} \dot{\theta}=\frac{f b \Omega}{V} \sum_{s=0}^{n} q_{s}^{\prime} z^{2 s+1},
\end{gathered}
$$

where we have put $\cos \theta=1$,

$$
e_{s}^{\prime}=b^{2 s+1} e_{s}, \quad q_{s}^{\prime}=b^{2 s+1} q_{s}, \quad s=0,1, \ldots, n .
$$

The angular velocity $\Omega$ is related to the forward speed $V$. It can be assumed, without appreciable error, that

$$
V=\Omega r,
$$

where $r$ is the radius of contact of the wheel with the rail when the axle is perfectly centered on the track; in other words, $r$ is a constant.

Next, we define some parameters and two functions:

$$
\begin{aligned}
& \Gamma=f k / m V, \quad \Sigma=2 m b^{2} / I k, \quad h(z)=\frac{g}{2 b} \sum_{s=0}^{n} e_{s}^{\prime} z^{2 s+1}, \\
& p(z)=\frac{f b}{\operatorname{Ir}} \sum_{s=0}^{n} q_{s}{ }^{\prime} z^{2 s+1}, \quad A=f k / m b .
\end{aligned}
$$

Using these, Eqs. (2.12)-(2.13) become

$$
\ddot{z}+\Gamma \dot{z}+h(z)=A \theta, \quad \ddot{\theta}+\Gamma \Sigma \dot{\theta}=p(z) .
$$

The above set differs from that of Brann [8] in two significant ways. First of all, we have let 
$r_{1}-r_{2}$ be a polynomial in $y$ rather than be linear in it. Secondly, in addition to changing $y$ to a non-dimensional parameter, Brann changes the time scale to $\tau=V t / b$. Since one is interested in finding the effect of the speed $V$ on the system, such an alteration of the time scale is not permissible. Indeed, the inability to justify Brann's results theoretically is one of the major reasons for the present re-examination of his problem.

3. Existence of periodic bifurcating solutions. Let us introduce the notation:

$$
z=x_{1}, \dot{z}=x_{2}, \quad \theta=x_{3}, \dot{\theta}=x_{4} \text {. }
$$

Then (2.17) become:

$$
\begin{gathered}
\dot{x}_{1}=x_{2}, \dot{x}_{2}=A x_{3}-\Gamma x_{2}-h\left(x_{1}\right), \\
\dot{x}_{3}=x_{4}, \quad \dot{x}_{4}=p\left(x_{1}\right)-\Gamma \Sigma x_{4} .
\end{gathered}
$$

The constants $\Sigma$ and $A$ are both positive, and the parameter $\Gamma>0$ as well.

Short reflection will convince the reader that $\tan \delta_{2}-\tan \delta_{1}$ increases with $y$, while $r_{1}$ $r_{2}$ decreases with $y$. Hence we obtain

$$
\begin{gathered}
h(0)=0, h_{1}=h^{\prime}(0)>0, h^{\prime \prime}(0)=0, \\
p(0)=0,-p_{1}=p^{\prime}(0)<0, p^{\prime \prime}(0)=0 .
\end{gathered}
$$

Let the system (3.2) be rewritten as

$$
\dot{\mathbf{x}}=\hat{\mathrm{F}}(\mathbf{x}, \Gamma) \text {. }
$$

Clearly, $\mathrm{F}(0, \Gamma)=0$ for all $\Gamma>0$. The converse is clearly true, or $\mathrm{F}(\mathbf{x}, \Gamma)=0 \Rightarrow \mathbf{x} \equiv 0$. Therefore we have established that:

The system

$$
\hat{F}(x, \Gamma)=0, \quad \Gamma>0
$$

has the unique critical point $\mathrm{x}=0$.

Now consider the gradient $\nabla_{\mathbf{x}} \hat{F}$ at $\mathbf{x}=0$. This has the matrix form

$$
\left[\nabla_{\mathbf{x}} \hat{\mathrm{F}}(0, \Gamma)\right]=\left[\begin{array}{cccc}
0 & 1 & 0 & 0 \\
-h_{1} & -\Gamma & A & 0 \\
0 & 0 & 0 & 1 \\
-p_{1} & 0 & 0 & -\Gamma
\end{array}\right] .
$$

The characteristic equation of the above matrix is equivalent to the polynomial equation:

$$
\mu^{4}+\Gamma(1+\Sigma) \mu^{3}+\left(\Gamma^{2} \Sigma+h_{1}\right) \mu^{2}+h_{1} \Gamma \Sigma \mu+p_{1} A=0 .
$$

Let us now seek the value of $\Gamma$, say $\Gamma_{0}$, so that (3.7) has the following four roots:

$$
\mu_{1} \pm i \mu_{2}, \mu_{1} \neq 0, \pm i \omega_{0}, \omega_{0}>0 \text {. }
$$

It is easy to show that

$$
\begin{gathered}
\mu_{1}=-\Gamma(1+\Sigma) / 2<0, \\
\mu_{2}{ }^{2}=(1+\Sigma)\left[h_{1}{ }^{2} \Sigma-A p_{1}(1-\Sigma)^{2}\right] / 4 h_{1} \Sigma^{2}, \\
\omega_{0}{ }^{2}=h_{1} \Sigma /(1+\Sigma),
\end{gathered}
$$


provided

$$
\Gamma_{0}^{2}=\frac{A p_{1}(1+\Sigma)^{2}-h_{1}{ }^{2} \Sigma}{h_{1} \Sigma^{2}(1+\Sigma)^{2}} .
$$

Since $\Gamma_{0}{ }^{2}>0,(3.11)$ shows that the constants $A, p_{1}, \Sigma$ and $h_{1}$ are subject to the inequality

$$
A p_{1}(1+\Sigma)^{2}>h_{1}^{2} \Sigma
$$

which will be assumed to hold in what follows.

Now let us redefine $\mathrm{F}(\mathbf{x}, \Gamma)$, when $\Gamma$ is close to $\Gamma_{0}$, as a function of $\epsilon=\Gamma-\Gamma_{0}$. That is:

$$
\mathrm{F}(x, \epsilon)=\hat{\mathrm{F}}\left(\mathbf{x}, \Gamma_{0}+\epsilon\right) .
$$

Clearly, the critical point of $\mathbf{F}(\mathbf{x}, \epsilon)=0$ is $\boldsymbol{a}^{\epsilon} \equiv \mathbf{x}=0$ which is needed to define $\mathbf{A}^{\epsilon}$, as in Poore [4]. This given by:

$$
\mathbf{A}^{\epsilon} \equiv \nabla_{\mathbf{x}} \mathrm{F}\left(\boldsymbol{a}^{\epsilon}, \epsilon\right)=\nabla_{\mathbf{x}} \mathrm{F}(0, \epsilon)
$$

The vector form of $\mathbf{F}(\mathbf{x}, \boldsymbol{\epsilon})$ is:

Hence,

$$
[\mathrm{F}(\mathbf{x}, \epsilon)]=\left[\begin{array}{ll}
x_{2} & \\
-h\left(x_{1}\right) & -\left(\Gamma_{0}+\epsilon\right) x_{2}+A x_{3} \\
x_{4} & \\
p\left(x_{1}\right) & -\left(\Gamma_{0}+\epsilon\right) \Sigma x_{4}
\end{array}\right] .
$$

$$
\left[\mathbf{A}^{\epsilon}\right]=\left[\begin{array}{cccc}
0 & 1 & 0 & 0 \\
-h_{1} & -\left(\Gamma_{0}+\epsilon\right) & A & 0 \\
0 & 0 & 0 & 1 \\
-p_{1} & 0 & 0 & -\left(\Gamma_{0}+\epsilon\right) \Sigma .
\end{array}\right]
$$

The equation $\operatorname{det}\left(\mathbf{A}^{\epsilon}-\mu 1\right)=0$ is equivalent to:

$$
\mu^{4}+\left(\Gamma_{0}+\epsilon\right)(1+\Sigma) \mu^{3}+\left(h_{1}+\Sigma\left(\Gamma_{0}+\epsilon\right)^{2}\right) \mu^{2}+h_{1} \Sigma\left(\Gamma_{0}+\epsilon\right) \mu+A p_{1}=0 .
$$

Linearization with respect to $\epsilon$ leads to

$$
\mu^{4}+\left(\Gamma_{0}+\epsilon\right)(1+\Sigma) \mu^{3}+\left(h_{1}+\Sigma\left(\Gamma_{0}^{2}+2 \Gamma_{0} \epsilon\right)\right) \mu^{2}+h_{1} \Sigma\left(\Gamma_{0}+\epsilon\right) \mu+A p_{1}=0 .
$$

Now suppose that the eigenvalues of $\mathbf{A}^{\epsilon}$ are:

$$
\mu_{1}+\epsilon \mu_{1}^{\prime} \pm i\left(\mu_{2}+\epsilon \mu_{2}^{\prime}\right), \epsilon \alpha^{\prime} \pm i\left(\omega_{0}+\epsilon \omega^{\prime}\right),
$$

where $\mu_{1}, \mu_{2}$ and $\omega_{0}$ are those in (3.8). Comparing the quartic polynomial in $\mu$ obtained by using (3.19) with (3.17), one derives the following:

$$
\begin{gathered}
-2\left(\alpha^{\prime}+\mu_{1}{ }^{\prime}\right)=1+\Sigma, \\
\mu_{1} \mu_{1}{ }^{\prime}+\mu_{2} \mu_{2}{ }^{\prime}+\omega_{0} \omega^{\prime}+2 \alpha^{\prime} \mu_{1}=\Gamma_{0} \Sigma, \\
-2 \mu_{1}{ }^{\prime} \omega_{0}{ }^{2}-4 \mu_{1} \omega_{0} \omega^{\prime}-2 \alpha^{\prime}\left(\mu_{1}{ }^{2}+\mu_{2}^{2}\right)=h_{1} \Sigma, \\
\omega_{0}\left(\mu_{1} \mu_{1}^{\prime}+\mu_{2} \mu_{2}{ }^{\prime}\right)+\omega^{\prime}\left(\mu_{1}{ }^{2}+\mu_{2}^{2}\right)=0 .
\end{gathered}
$$

A straightforward calculation shows that

$$
\alpha^{\prime}=2 \mu_{1} \Gamma_{0} \Sigma \omega^{2} /\left\{\left[\omega_{0}^{2}-\left(\mu_{1}^{2}+\mu_{2}^{2}\right)\right]^{2}+4 \mu_{1}^{2} \omega_{0}^{2}\right\} .
$$


Since $\mu_{1}<0$, and the other quantities are all greater than 0 ,

$$
\alpha^{\prime}<0 \text {. }
$$

It is clear that our calculations satisfy all the hypothesis of the existence theorem (Thm. 2.3) of Poore [4] and thus a periodic solution of

$$
\dot{\mathbf{x}}=\mathrm{F}(\mathbf{x}, \epsilon(\psi))
$$

exists near $\epsilon=0$. The period is $(1+\psi \eta(\psi)) T$, where $T=2 \pi N / \omega_{0}$, where $N$ is any positive integer. For the determination of $\eta=\eta(\psi), \epsilon=\epsilon(\psi)=\psi \delta(\psi)$, the reader is referred to Poore [4], though we shall compute the sign of $\delta^{\prime}(0)$ to determine the stability of the bifurcated periodic orbit next.

4. Stability of the bifurcated orbit. The algorithm for determining the stability of the periodic orbit has been given by Poore [4] and this algorithm reduces this question to the determination of the sign of $\alpha^{\prime} \delta^{\prime}(0)$ and the real parts of the remaining eigenvalues of $\mathbf{A}^{0}$. Since we have, in (3.9), shown that the real parts of the eigenvalues $\mu_{1} \pm i \mu_{2}$ are negative, from Poore's [4] Theorem 3.1 it follows that the bifurcated periodic orbit will be asymptotically orbitally stable if $\alpha^{\prime} \delta^{\prime}(0)>0$.

To determine the sign of this quantity, we note from Theorem 4.1 of [4] that

$$
\begin{aligned}
8 \alpha^{\prime} \delta^{\prime}(0) & =\operatorname{Re}\left\{-u_{l} \frac{\partial^{3} F^{\prime}}{\partial x_{j} \partial x_{k} \partial x_{p}} v_{j} v_{k} \bar{v}_{p}+2 u_{l} \frac{\partial^{2} F^{l}}{\partial x_{j} \partial x_{k}} v_{j}\left(A^{0}\right)_{k r}{ }^{-1} \frac{\partial^{2} F^{r}}{\partial x_{p} \partial x_{q}} v_{p} \bar{v}_{q}\right. \\
& \left.+u_{l} \frac{\partial^{2} F^{\prime}}{\partial x_{j} \partial x_{k}} \bar{v}_{j}\left(A^{0}-2 i \omega_{0} I\right)_{k r}{ }^{-1} \frac{\partial^{2} F^{r}}{\partial x_{p} \partial x_{q}} v_{p} v_{q}\right\} .
\end{aligned}
$$

Here, the higher gradients of $F$ are:

$$
\begin{aligned}
& \frac{\partial^{2} F^{\prime}}{\partial x_{j} \partial x_{k}}=\left.\frac{\partial^{2} F^{\prime}}{\partial x_{j} \partial x_{k}}\left(x_{m}, \epsilon\right)\right|_{x_{m}=0, \epsilon=0}, \\
& \frac{\partial^{3} F^{\prime}}{\partial x_{j} \partial x_{k} \partial x_{p}}=\left.\frac{\partial^{3} F^{\prime}\left(x_{m}, \epsilon\right)}{\partial x_{j} \partial x_{k} \partial x_{p}}\right|_{x_{m}=0, \epsilon=0} .
\end{aligned} .
$$

From (3.15) and the assumptions (3.3), it follows that

$$
\begin{gathered}
\frac{\partial^{2} F^{l}}{\partial x_{j} \partial x_{k}}=0, \\
\frac{\partial^{3} F^{2}}{\partial x_{1} \partial x_{1} \partial x_{1}}=-h^{\prime \prime \prime}(0), \quad \frac{\partial^{3} F^{4}}{\partial x_{1} \partial x_{1} \partial x_{1}}=p^{\prime \prime \prime}(0), \quad \text { others zero . }
\end{gathered}
$$

Moreover, $\mathbf{u}$ and $\mathbf{v}$ are respectively the normalized left and right eigenvectors of $\mathbf{A}^{0}$ corresponding to $+i \omega_{0}$. That is,

$$
\mathbf{A}^{0} \mathbf{v}=i \omega_{0} \mathbf{v}, \quad \mathbf{u A}^{0}=i \omega_{0} \mathrm{u}, \quad \mathrm{uv}=1,
$$

and $\bar{v}$ is the conjugate of $\mathbf{v}$. It is clear from the foregoing that we have to compute the two eigenvectors $\mathbf{u}$ and $\mathbf{v}$ subject to the normalization condition $u_{i} v_{i}=1$ and find the sign of the real part of the expression

$$
\left(h^{\prime \prime \prime}(0) u_{2}-p^{\prime \prime \prime}(0) u_{4}\right) v_{1}^{2} \bar{v}_{1}
$$

to answer the question concerning the stability of the bifurcated orbit. It is extremely 
interesting that $\left(h^{\prime \prime \prime}(0) u_{2}-p^{\prime \prime \prime}(0) u_{4}\right)$ must be nonzero for this answer to be given. Let us compute $\mathbf{v}$ and $\mathbf{u}$ next and obtain

$$
\begin{gathered}
{[\mathbf{v}]=\left[\begin{array}{l}
1 \\
i \omega_{0} \\
h_{1}+i \omega_{0}\left(\Gamma_{0}+i \omega_{0}\right) \\
i \omega_{0} h_{1}-\left(\Gamma_{0}+i \omega_{0}\right) \omega_{0}^{2}
\end{array}\right] v_{1}, \quad v_{1} \neq 0,} \\
{[\mathbf{u}]=u_{4}\left[i\left(\Gamma_{0}+i \omega_{0}\right) \omega_{0}\left(\Gamma_{0} \Sigma+i \omega_{0}\right), i \omega_{0}\left(\Gamma_{0} \Sigma+i \omega_{0}\right), A\left(\Gamma_{0} \Sigma+i \omega_{0}\right), 1\right], \quad u_{4} \neq 0 .}
\end{gathered}
$$

The normalization condition $u_{i} v_{i}=1$ means that

$u_{4}=\left[\left(\Gamma_{0} \sigma+i \omega_{0}\right)\left(A h_{1}-(2+A) \omega_{0}^{2}+i \omega_{0} \Gamma_{0}(1+A)\right)+i \omega_{0} h_{1}-\omega_{0}^{2}\left(\Gamma_{0}+\omega_{0}\right)\right]^{-1}$,

on taking $v_{1}=1$, which is permissible. Therefore, the bifurcated periodic orbit is stable if

$\operatorname{Re}\left\{\frac{\left(\omega_{0}^{2} h^{\prime \prime \prime}(0)+p^{\prime \prime \prime}(0)-i \omega_{0} h^{\prime \prime \prime}(0) \Gamma_{0} \mathbf{z}\right)}{\omega_{0}^{2}\left(\Gamma_{0}+i \omega_{0}\right)-i \omega_{0} h_{1}-\left(\Gamma_{0} \mathbf{z}+i \omega_{0}\right)\left(A h_{1}-(2+A) \omega_{0}{ }^{2}+i \omega_{0} \Gamma_{0}(1+A)\right)}\right\}>0$

and unstable if $\operatorname{Re}\{\cdot\}<0$.

Incidentally, stability means that $\delta^{\prime}(0)<0$, since $\alpha^{\prime}<0$. Therefore the periodic orbit bifurcates to the left of $\Gamma_{0}$ [4]. Since $\Gamma$ decreases as the speed $V$ increases, it is clear that as the speed of the train is increased beyond $V_{0}$ corresponding to $\mathrm{I}_{0}$, the periodic orbit bifurcates at a value of $0<\mathrm{I}<\mathrm{I}_{0}$, or for a speed $V>V_{0}$.

Turning to Brann's paper [8], one may obtain the numerical values for $\mathrm{I}_{0}, \omega_{0}, \mathbf{\Sigma}, h_{1}$ and $A$ to verify the stability of the orbit. These are calculated from Appendix II of [8] to be:

$$
\begin{gathered}
b=28 \text { in }, r=18 \mathrm{in} ; \\
f=\mathrm{mg} / 2, I=m b^{2}, g=32.2 \mathrm{ft} / \mathrm{sec}^{2}, k \doteq 2 \\
\Sigma=\frac{2 m b^{2}}{I k}=\frac{2}{k} \doteq 1, A=\frac{f k}{m b}=\frac{g k}{2 b} \doteq 13.8, h_{1} \doteq 0.69, \\
p_{1} \doteq \frac{0.05 g}{r}=1.073 / \mathrm{sec}^{2}, \omega_{0}^{2}=0.345 / \mathrm{sec}^{2}, \mathrm{I}_{0}^{2}=21.29 / \mathrm{sec}^{2} .
\end{gathered}
$$

Therefore the forward speed $V_{0}$ at which the periodic orbit bifurcates is given by

$$
V_{0}=\frac{f k}{m \Gamma_{0}}=\frac{g k}{2 \Gamma_{0}} \doteq \frac{g}{\Gamma_{0}}=6.98 \mathrm{ft} / \mathrm{sec} . \doteq 7.62 \mathrm{Kph} .
$$

Brann [8] assumes that $p^{\prime \prime \prime}(0)=0$ and that $h^{\prime \prime \prime}(0)>0$. The condition (4.10) now becomes:

Is $\operatorname{Re}\left\{\frac{\left(\omega_{0}{ }^{2}-i \omega \Gamma_{0} \Sigma\right)}{\omega_{0}{ }^{2}\left(\Gamma_{0}+i \omega_{0}\right)-i \omega_{0} h_{1}-\left(\Gamma_{0} \Sigma+i \omega_{0}\right)\left(A h_{1}-(2+A) \omega_{0}{ }^{2}+i \omega_{0} \Gamma_{0}(1+A)\right)}\right\} \gtrless 0$ ?

A straightforward calculation shows that the left side is equal to:

$$
\operatorname{Re}\left\{\frac{0.345-i 2.71}{6.37-i 187.67}\right\}
$$


and this is indeed positive. Therefore the bifurcated periodic orbit is stable and the bifurcation occurs to the left of $\Gamma_{0}$.

5. Concluding remarks. As can be seen, in the derivation of the system (3.2), a number of assumptions, equivalent to taking $\theta<<1$, were made. Therefore, it is physically meaningless to think of (3.2) as representing a realistic model of finite-amplitude oscillations of the wheel set. Nevertheless, from an analytical point of view, the bifurcated periodic orbit of the system (3.2) can be shown to possess either an unbounded amplitude leading to derailment, or an unbounded period, or a combination of both. These points will be dealt with in a later paper, using a fully nonlinear model.

\section{REFERENCES}

[1] F. W. Carter, Railway electric traction, Arnold (London), 1922

[2] E. Hopf, Abzweigung einer periodischer Lösung von einer stationären Lösung eines Differentialsystems, Ber. Verh. Sächs. Akad. Wiss. Leipzig. Math. Nat. KI. 94, 1-22 (1942) (see also the translation in [5])

[3] K. O. Friedrichs, Advanced ordinary differential equations, Gordon and Breach (New York), 1965

[4] A. B. Poore, On the theory and application of the Hopf-Friedrichs bifurcation theory, Arch. Ratl. Mech. A nal. 60, 371-393 (1976)

[5] J. E. Marsden and M. McCracken, The Hopf bifurcation and its applications, Springer-Verlag (New York), 1976

[6] L. Cesari, Functional analysis and Galerkin's method, Mich. Math. J. 11, 385-418 (1964)

[7] J. K. Hale, Applications of alternative problems, Lecture Notes, Center for Dynamical Systems, Brown University, Providence, 1971

[8] R. P. Brann, Some aspects of the hunting of a railway axle, J. Sound. Vib. 4, 18-32 (1966)

[9] F. W. Carter, On the action of a locomotive driving wheel, Proc. Roy. Soc. Lond. A 112 151-157 (1926)

[10] J. J. Kalker, Survey of mechanics of contact between solid bodies, Zeit. angew. Math. Mech., 57, T3-T17 (1977) 